\title{
Anterior membrane dystrophy following cataract extraction
}

\author{
JOHN D. BRODRICK \\ From the University of Texas Health Science Center, Dallas, USA
}

\begin{abstract}
SUMMARY This paper describes a patient with previously normal corneas who underwent bilateral cataract surgery. This was followed by acute dehiscence of the epithelium and the eventual appearance of fingerprint lines, microcysts, and map-like areas typical of Cogan's microcystic dystrophy. Light and electron microscopy showed the presence of epithelial microcysts and a seam of subepithelial material like basement membrane. It is suggested that this patient represents a rapid transition from apparent normality to an extensive anterior membrane dystrophy, precipitated by operation for cataract.
\end{abstract}

A unique corneal complication of cataract surgery is presented in this paper, which describes the bilateral acute onset of an anterior membrane dystrophy. Fingerprint lines of the cornea were first described by Guerry as fine wavy lines with whorllike contours located within the corneal epithelium and best seen by retroillumination (Guerry, 1950). He noted similar lines in keratoconus and advanced Fuchs's dystrophy. His clinical observations were confirmed and supplemented by subsequent authors, who demonstrated histologically a splitting of Bowman's membrane (DeVoe, 1962; Kaufman and Clowers, 1966). Recent authors have included finger-print, net, and bleb dystrophies with Cogan's microcystic dystrophy (Cogan et al., 1964) in a complex of interrelated superficial corneal disorders, the anterior membrane dystrophies (Bron and Brown, 1971; Trobe and Laibson, 1972). It has been suggested that anomalies of the basement membrane are the prime cause of both the fingerprint lines and the epithelial microcysts (Cogan et al., 1974; Rodrigues et al., 1974).

Fingerprint striae have recently been described both as an idiopathic dystrophic form (Brodrick et al., 1974a) and as an accompaniment of herpes simplex keratitis (Brodrick et al., 1976). There is only one previous report of the condition occurring in an aphakic patient (Rodrigues et al., 1974), but no mention has been made of the explosive onset and progressive course seen in the case discussed here. This contrasts with a recent report of 5 patients

Address for reprints: Professor John D. Brodrick, University of Texas, Department of Ophthalmology, Southwestern Medical School, 5323 Harry Hines Boulevard, Dallas, Texas 75235 , USA with Cogan's microcystic dystrophy who had intraocular surgery without any unfavourable effects except for mild progression in one patient (Luxenberg et al., 1975).

\section{Case report}

A 62-year-old Caucasian woman with no previous history of ocular or general disease was first seen in 1970 with failing vision due to bilateral cataracts. Corneal examination at that time was normal. By 1972 her vision had deteriorated considerably and lens extraction was recommended. Apart from the cataracts, preoperative examination of her eyes revealed no other abnormality.

In September 1972 a routine intracapsular lens extraction was carried out with an uneventful postoperative period. Three weeks later she developed a sensation of a foreign body in the left eye which rapidly became intensely painful, accompanied by extreme photophobia and lacrimation. Examination showed swelling of the left eyelids, intense blepharospasm, and profuse tearing. There was marked conjunctival hyperaemia, and the left cornea appeared hazy.

Slit-lamp examination showed that the lower half of the cornea was covered by circumferential whorls which closely resembled a thumbprint (Fig. 1). At high magnification these whorls were seen to consist of multiple small ridges in oedematous epithelium which was heaped up into folds. Moreover the whole of the affected area was slightly mobile and was dehiscing from the underlying basal lamina and stroma. No staining was noted, but the tear film was broken up by the epithelial ridges. Bowman's 


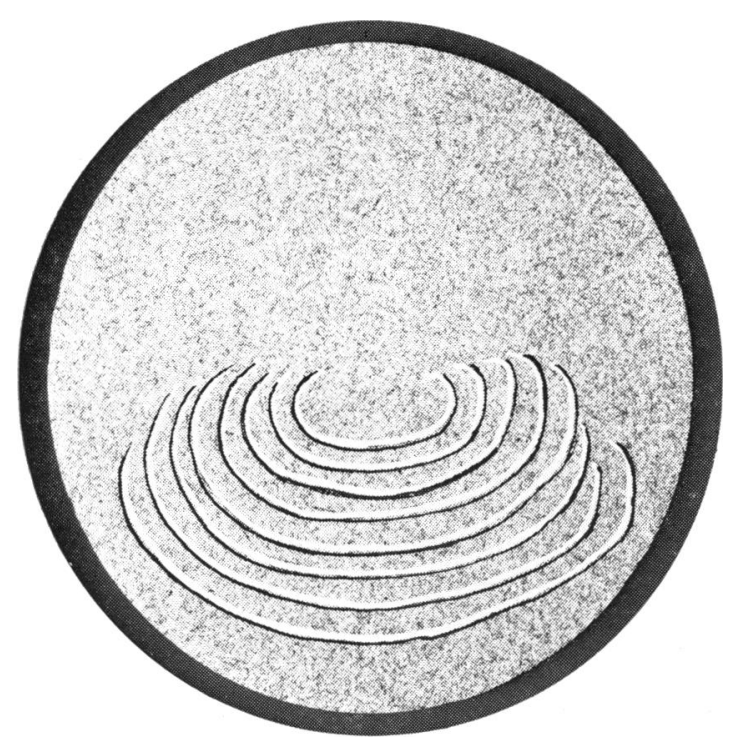

Fig. 1 Drawing of cornea to illustrate the overall distribution of the epithelial whorls

layer appeared normal, and there was no evidence of stromal oedema. The stroma measured $0.5 \mathrm{~mm}$ with a normal endothelium and Descemet's membrane. The anterior chamber showed no significant flare or cells and the vitreous face was intact and well back. Intraocular pressure was $18 \mathrm{mmHg}$ by applanation tonometry. Corneal sensation was greatly reduced.

Treatment was continued with atropine and corticosteroid drops and admission was arranged for epithelial debridement $\mathbf{3}$ days later. In this short time, however, all striae and affected epithelium were shed and her symptoms completely abated. Biomicroscopy now showed that the whorls had been replaced by scattered epithelial microcysts and greyish map-like areas consistent with the diagnosis of Cogan's microcystic dystrophy (Fig. 2). The cornea was otherwise normal apart from the decreased sensation.

Since then she has experienced intermittent episodes of discomfort and foreign body sensation, particularly on awakening in the morning, tending to alleviate as the day progressed. By January 1973 in addition to the features of Cogan's microcystic dystrophy some fine fingerprint striae had appeared in the nasal periphery (Fig. 3). Throughout this period the right cornea was entirely normal to clinical examination.

In May 1973 an uncomplicated intracapsular lens extraction was performed on the right eye with an uneventful postoperative period. Twelve weeks afterwards severe pain and discomfort developed in the right eye. Examination revealed swelling of the lids, intense blepharospasm, lacrimation, and photophobia. The conjunctiva was hyperaemic and the lower half of the cornea appeared hazy. Slit-lamp examination showed that the upper half of the cornea was normal; the lower half was oedematous and heaped up into striae, the appearances being identical to those previously seen in the left cornea. The epithelium was extremely mobile, and, as in the left cornea, though no stain was present the tear film was broken up by the epithelial striae. Corneal sensation was reduced. There was no apparent abnormality of Bowman's layer, the

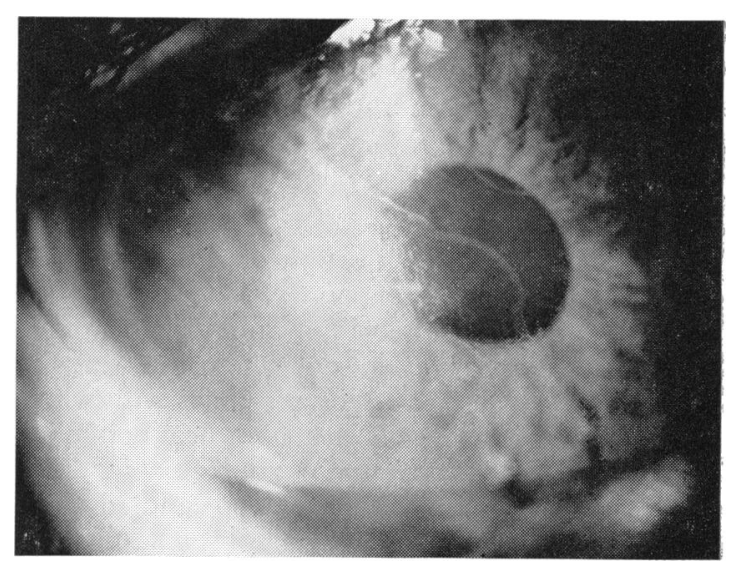

Fig. 2 Photograph of cornea showing the large map-like areas in the centre $(\times 1)$

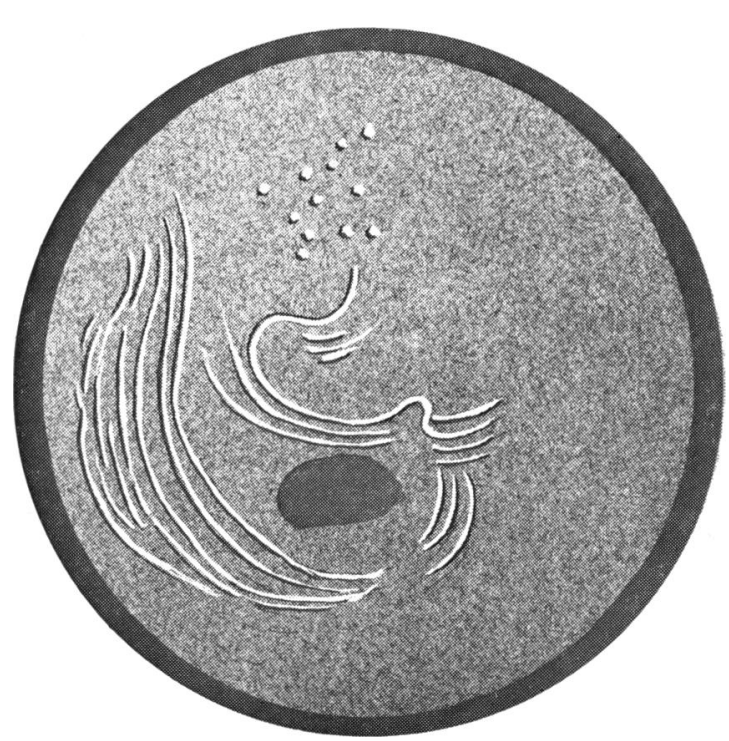

Fig. 3 Drawing of cornea to illustrate the distribution of map-like areas, fingerprint striae, and microcysts 
stroma, Descemet's membrane, or the endothelium.

The anterior chamber was quiet, the vitreous face intact and well back, and the intraocular pressure was $19 \mathrm{mmHg}$ by application tonometry. The affected epithelium was removed immediately under topical anaesthesia. The specimen was divided into 2 ; one half was fixed in formalin and embedded in paraffin for conventional microscopy, the other half was fixed in cold $2.5 \%$ gluteraldehyde, washed, and postfixed in $1 \%$ osmium tetroxide and embedded in Araldite for electron microscopy.

Her subsequent progress has been satisfactory, with a corrected vision of 20/25 in each eye. She still has intermittent attacks of discomfort and a foreign body sensation affecting both eyes, usually when she wakes in the morning. They tend to improve as the day progresses. There have been no further attacks of intense pain.

Examination in 1974 showed the following corneal appearances. In the right central cornea a greyish, map-like area with fine linear borders was visible, lying in the deep aspect of the epithelium. Some fine fingerprint striae were seen in the upper nasal and lower temporal periphery, and scattered epithelial microcysts were evident both centrally and peripherally. The microcysts and fingerprint striae were best seen by retroillumination and showed reverse illumination, denoting increased optical density of their contents (Graves, 1924). The left cornea showed numerous whorled fingerprint striae mainly in the nasal half of the cornea. Some scattered epithelial microcysts were observed in the supranasal quadrant, and a greyish map-like area could be seen just below the centre of the cornea. The striae and microcysts were best seen by retroillumination and showed reverse illumination as in the right eye. Schirmer's test with local anaesthesia revealed decreased lacrimal secretion, $4 \mathrm{~mm}$ in 5 minutes in the right eye, $3 \mathrm{~mm}$ in 5 minutes in the left eye. The tear film showed break-up over the area of the fingerprint striae, so that linear dry zones rapidly became apparent. The anterior corneal mosaic was interrupted in the areas of the epithelial changes. The microcysts showed no affinity for fluorescein. Corneal sensation assessed with monofilament silk of varying calibre was normal. In both eyes the corneal stroma was normal, with no visible abnormality of Bowman's layer, Descemet's membrane, or the endothelium. The anterior chambers were deep and quiet, the vitreous face intact and well back, and intraocular pressure $19 \mathrm{mmHg}$ by applanation tonometry.

LIGHT MICROSCOPY

The specimen comprised epithelium, basal lamina, and some superficial stromal lamellae. The epithe- lium showed the normal 5-layered structure. The cellular morphology was essentially normal, with no evidence of pleomorphism, acanthosis, or acantholysis. There was no dysplasia, but scattered areas of spongiosis were noted. Epithelial microcysts of varying shape and size were located at different levels (Fig. 4). In some areas pale staining basal cells were observed. The microcysts contained a mixture of intensely basophilic coarse granules and a pale blue, finely granular substance. The nuclei were pyknotic, and the intercellular debris was characteristic of degenerating epithelial cells.

A thin seam of moderately basophilic, homogeneous material was present deep to the apparently normal basal lamina. In some sections small projections from this seam appeared to intrude into the epithelium. The subepithelial material was periodic acid Schiff negative (Fig. 5).

\section{ELECTRON MICROSCOPY}

The epithelial microcysts presented a typical appearance with microvillous projections into a cavity containing a variety of degenerating organelles and nuclear material (Fig. 6). The subepithelial seam consisted of a matrix of closely-packed granules $80 \AA$ in diameter containing randomly interspersed fibrillae $170 \AA(17 \mathrm{~nm})$ in width, banded at $125-\AA$ intervals. In some areas this seam projected up into the epithelium. The basement membrane and Bowman's layer were of normal thickness and contour and the superficial stromal lamellae were normal.

\section{Discussion}

This case is of interest because it represents a transition from a clinically normal asymptomatic

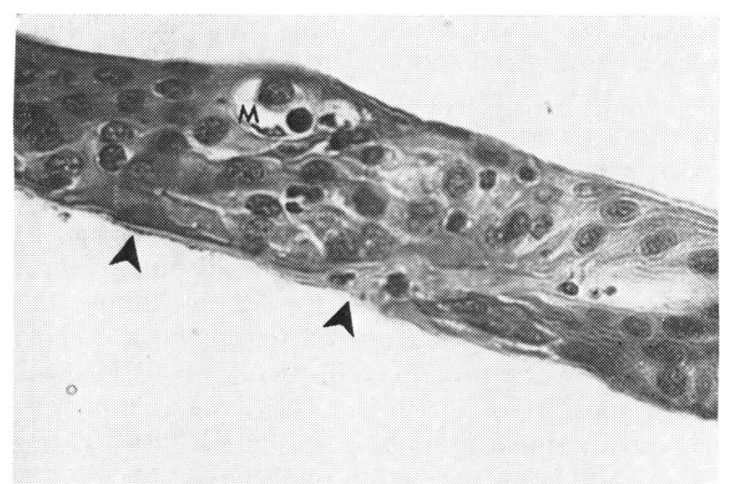

Fig. 4 Section of epithelium showing marked dysplasia, a superficial microcyst $(\mathrm{M})$, and a layer of abnormal subepithelial tissue intruding up into the epithelium (Masson, $\times 500$ ) 


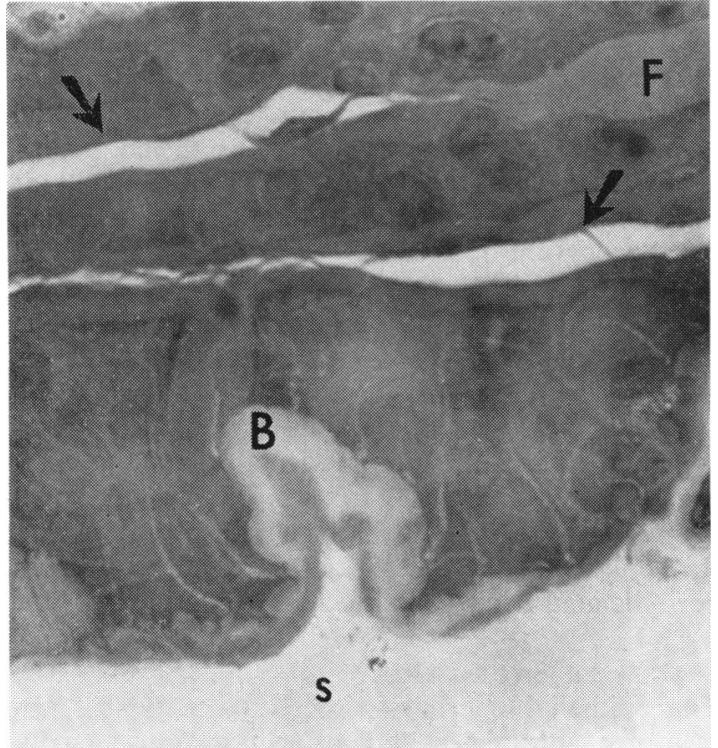

Fig. 5 Section of epithelium showing subepithelial seam of abnormal tissue (S) invaginating the grossly thickened basement membrane (B) up into the epithelium. Clear spaces (arrowed) are artefacts, but possibly represent a plane of cleavage caused by fingerprint line (F) (Masson, $\times 1750$ )

cornea through an acute episode of epithelial dehiscence to an established anterior membrane dystrophy of the Cogan's microcystic and fingerprint variety.

Epithelial microcysts are a feature of many corneal disorders, including the recurrent erosion syndrome (Tripathi and Bron, 1972), Meesman's epithelial dystrophy (Kuwabara and Cicarelli, 1964), and Cogan's microcystic dystrophy (Guerry, 1965). Their presence has also been noted in a complex of interrelated superficial corneal disorders (Bron and Brown, 1971) now regarded as a distinct group, the anterior membrane dystrophies. The microcysts described in these various conditions appear histologically identical (Rodrigues et al., 1974; Brodrick et al., 1974, 1976). Although the precise mechanism of the microcyst formation is still in dispute, several theories exist to account for it, including the inhibited migration theory of Cogan et al. (1974) and the inverted basal cell theory of Rodrigues (Rodrigues et al., 1974). They may, however, represent a much more basic entity (Brodrick $e t$ al., 1974), and it would seem reasonable to suggest that the cornea, whose unique and fundamental property is its transparency, has by virtue of its avascular nature only a limited number of inherent pathological responses. It has been suggested that the microcyst reaction represents a process whereby the corneal epithelium secludes and deletes degenerating cells, a type of epithelial phagocytosis, although a true phagosome is probably not formed (Brodrick et al., 1974).

The seam of subepithelial material which probably results from disordered metabolism of the basal epithelial cells readily explains the defective epithelial adhesion. A similar seam has been documented as the basis of fingerprint dystrophy (Brodrick et al., 1974).

It has frequently been commented that the superficial corneal disorders represent a broad spectrum of probably interrelated disorders, and a search has been made for a common pathogenesis which may not, however, exist. It is more probable that a variety of aetiological agents, including a true dystrophic process in the basal cells, can pursue a final common path to produce a similar ultrastructural and clinical appearance.

The importance of the present case is that it represents a rapid evolution, probably precipitated by surgery, from clinically normal cornea to the typical appearances of Cogan's microcystic dystrophy with fingerprint lines. The intermediate phase of acute epithelial dehiscence is similar to the

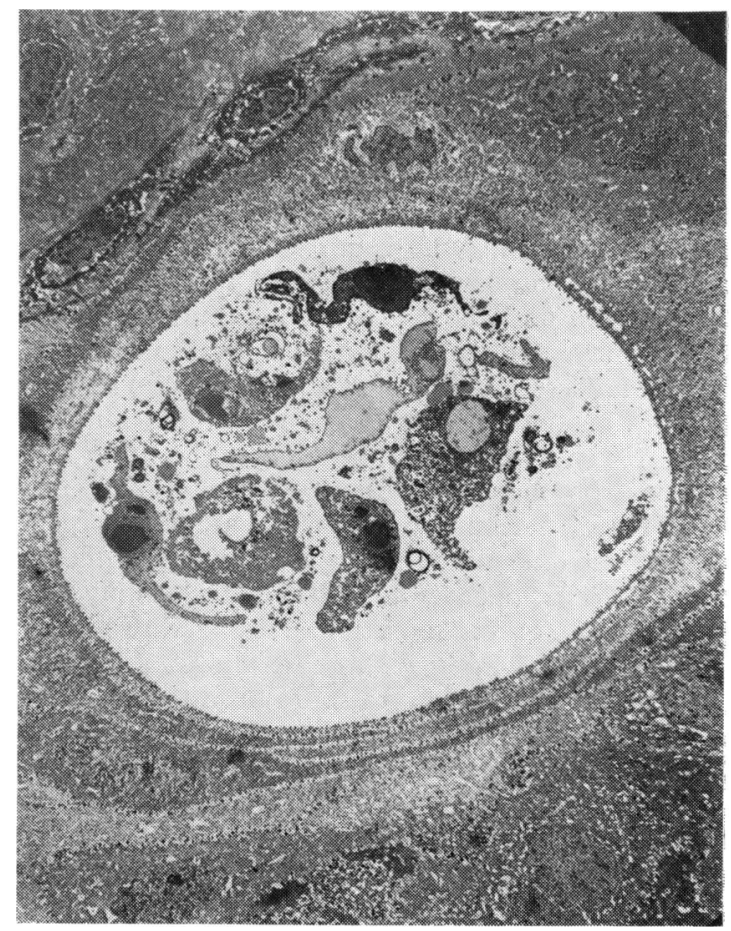

Fig. 6 Electronmicrograph showing epithelial microcyst with microvillous lining and degenerating cellular debris in the cyst cavity (uranyl acetate and lead citrate, $\times 800$ ) 
superficial linear keratitis described by Spicer and Greeves (1916). An unusual feature was the localisation entirely in the lower half of each cornea. One might have expected these changes to occur in the upper half adjacent to the cataract incision, where the effects of surgical trauma and the interference with nutrition and sensation would be maximal. It is difficult to explain this distribution.

This case, apart from its unusual features, adds further support to the concept that a series of dissimilar epithelial reactions may be transitional states in a spectrum of related disorders. The fundamental disorder common to the group is probably a disturbance in the metabolism of the basal epithelial cells. This results in the formation of the subepithelial material responsible for epithelial adhesion problems and fingerprint lines and triggers off the formation of microcysts, enabling the cornea to degrade and extrude degenerating and damaged cells by a process which does not prejudice its normal avascularity and transparency.

\section{References}

Brodrick, J. D., Dark, A. J., and Peace, G. W. (1974). Fingerprint dystrophy of the cornea. Archives of Ophthalmology, 92, 483-489.

Brodrick, J. D., Dark, A. J., and Peace, G. W. (1976) Fingerprint lines of the cornea in herpes simplex keratitis. Annals of Ophthalmology, 8, 481-484.

Bron, A. J., and Brown, N. A. (1971). Some superficial corneal disorders. Transactions of the Ophthalmological Societies of the United Kingdom, 91, 13-29.

Cogan D. G., Donaldson, D., and Kuwabara, T. (1964). Microcystic dystrophy of the corneal epithelium. Trans- actions of the American Ophthalmologic Society, 62, 213225.

Cogan, D. G., Kuwabara, T., Donaldson, O. O., and Collins, E. (1974). Microcystic dystrophy of the cornea. Archives of Ophthalmology, 92, 470-474.

DeVoe, A. G. (1962). Certain abnormalities of Bowman's membrane with particular reference to fingerprint lines in the cornea. Transactions of the American Ophthalmologic Society, 60, 195-201.

Graves, B. (1924). A bilateral chronic affection of the endothelial face of the cornea of elderly persons with an account of the technical and clinical principles of its slitlamp observation. British Journal of Ophthalmology, 8, 502-544.

Guerry, D. (1950). Fingerprint-like lines in the cornea. American Journal of Ophthalmology, 33, 724-727.

Guerry, D. (1965). Observations on Cogan's microcystic dystrophy of the corneal epithelium. Transactions of the American Ophthalmologic Society, 63, 320-334.

Kaufman, H. E., and Clower, J. W. (1966). Irregularities of Bowman's membrane. American Journal of Ophthalmology, 61, 227-230.

Kuwabara, T., and Cicarelli, E. C. (1964). Meesman's corneal dystrophy. Archives of Ophthalmology, 71, 676682.

Luxenberg, M. N., Friedland, Beth R., and Holden, J. M. (1975). Superficial microcystic corneal dystrophy. Archives of Ophthalmology, 93, 107-110.

Rodrigues, M. M., Fine, B. S., Laibson, P. R., and Zimmerman, L. E. (1974). Disorders of the epithelium. Archives of Ophthalmology, 92, 475-482.

Spicer, W. T. N., and Greeves, R. A. (1916). On superficial linear keratitis together with an account of the pathological examination of two affected eyes. Ophthalmoscope, 14, 116-120.

Tripathi, R. C., and Bron, A. J. (1972). Ultrastructural study of non-traumatic recurrent erosion. British Journal of Ophthalmology, 56, 73-85.

Trobe, J. D., and Laibson, P. R. (1972). Dystrophic changes in the anterior cornea. Archives of Ophthalmology, 87, 378-382. 